\title{
Magnetic field perturbation of neural recording and stimulating microelectrodes
}

\author{
Francisco M Martinez-Santiesteban ${ }^{1}$, Scott D Swanson ${ }^{2}$, Douglas C Noll ${ }^{1}$ \\ and David J Anderson ${ }^{1,3}$ \\ ${ }^{1}$ Department of Biomedical Engineering, University of Michigan, Ann Arbor, MI 48109, USA \\ 2 Department of Radiology, University of Michigan, Ann Arbor, MI 48109, USA \\ ${ }^{3}$ Department of Electrical Engineering and Computer Science, University of Michigan, \\ Ann Arbor, MI 48109, USA \\ E-mail: sfmartin@umich.edu
}

Received 13 November 2006, in final form 7 February 2007

Published 26 March 2007

Online at stacks.iop.org/PMB/52/2073

\begin{abstract}
To improve the overall temporal and spatial resolution of brain mapping techniques, in animal models, some attempts have been reported to join electrophysiological methods with functional magnetic resonance imaging (fMRI). However, little attention has been paid to the image artefacts produced by the microelectrodes that compromise the anatomical or functional information of those studies. This work presents a group of simulations and MR images that show the limitations of wire microelectrodes and the potential advantages of silicon technology, in terms of image quality, in MRI environments. Magnetic field perturbations are calculated using a Fourierbased method for platinum (Pt) and tungsten (W) microwires as well as two different silicon technologies. We conclude that image artefacts produced by microelectrodes are highly dependent not only on the magnetic susceptibility of the materials used but also on the size, shape and orientation of the electrodes with respect to the main magnetic field. In addition silicon microelectrodes present better MRI characteristics than metallic microelectrodes. However, metallization layers added to silicon materials can adversely affect the quality of MR images. Therefore only those silicon microelectrodes that minimize the amount of metallic material can be considered MR-compatible and therefore suitable for possible simultaneous fMRI and electrophysiological studies. High resolution gradient echo images acquired at $2 \mathrm{~T}(\mathrm{TR} / \mathrm{TE}=$ $100 / 15 \mathrm{~ms}$, voxel size $\left.=100 \times 100 \times 100 \mu \mathrm{m}^{3}\right)$ of platinum-iridium $(\mathrm{Pt}-\mathrm{Ir}$, $90 \%-10 \%)$ and tungsten microwires show a complete signal loss that covers a volume significantly larger than the actual volume occupied by the microelectrodes: roughly 400 times larger for Pt-Ir and 180 for $\mathrm{W}$, at the tip of the microelectrodes. Similar MR images of a single-shank silicon microelectrode only produce a partial volume effect on the voxels occupied by the probe with less than $50 \%$ of signal loss.
\end{abstract}




\section{Introduction}

At present, brain mapping technologies to study brain function can be divided into two categories: those with high temporal resolution and those with high spatial resolution. Unfortunately, there is no single technique that includes both characteristics.

Some attempts have been reported to join the high temporal resolution and high spatial specificity obtained with extracellular electrophysiological recordings, using wire or carbon fibre microelectrodes, and the high volume coverage and good spatial resolution of the blood oxygenation level dependent (BOLD) phenomenon, obtained with fMRI (Logothetis et al 2001, Shyu et al 2004). Currently, the simultaneous use of these technologies requires some type of signal processing, magnet pulsing synchronization and special electronic devices. In addition, as explained by Schenck (1996), the magnetic susceptibility difference $(\Delta \chi)$ between tissue and metallic or carbon fibre microelectrodes causes significant image distortions that, while making very obvious the presence of the electrodes, may also compromise the anatomical information in regions around the electrodes or bias the fMRI results to regions remote from the actual electrode position.

In this work, we present a group of simulations and MR images that show the limitations, in terms of image quality, of common wire microelectrodes, and the advantages of silicon substrate electrode technology. The presented results may help potential users of simultaneous electrophysiology and fMRI, to have a more adequate selection of materials and a better understanding of MRI artefacts generated by microelectrodes. Moreover, the tools used to estimate the magnetic field perturbations can be used to estimate MRI artefacts produced by more detailed models and future designs.

\section{Materials and methods}

\subsection{Three-dimensional models}

First, we defined the spatial magnetic susceptibility distribution $\chi(\mathbf{r})$ of eight different models, as listed below. The values of magnetic susceptibility for the different materials were obtained from Schenck (1996) and Martínez-Santiesteban et al (2006).

(1) Infinite circular cylindrical wire made of platinum (Pt, $\chi=279 \mathrm{ppm})$.

(2) Same as the previous case but made of tungsten (W, $\chi=77.2 \mathrm{ppm}$ ).

(3) Same as case one but using a core of silicon ( $\mathrm{Si}, \chi=-4.2 \mathrm{ppm}$ ), with a layer of gold (Au, $\chi=-34 \mathrm{ppm}$ ) under an outer layer of Pt. The thickness of each layer was independently varied from 0 to $0.5 \mu \mathrm{m}$ in steps of $0.1 \mu \mathrm{m}$, keeping the total diameter of the model at $25 \mu \mathrm{m}$.

(4) A section of a platinum microelectrode with a total length of $500 \mu \mathrm{m}$ that includes a tapered tip of $250 \mu \mathrm{m}$ in length that ends with a diameter of $1.5 \mu \mathrm{m}$ at the tip.

(5) Same as the previous case but using tungsten.

(6) Same geometry as the previous two microwires, but this time using a core of silicon, an external $0.5 \mu \mathrm{m}$ thick layer of platinum on the top of an internal $0.5 \mu \mathrm{m}$ thick layer of gold, both covering the tapered tip of the electrode. This model is similar to a section of a single shank of the 100-shank electrode described by Campbell et al (1991), and known as the 'Utah probe' or 'Utah array'.

(7) A simplified model of a complete 100-channel Utah probe, purely made of silicon, excluding the metallic layers at the shanks, but including 100 square bonding pads at the base of the assembly, as shown in figure 1. The material used in the bonding pads of this particular probe has been reported in the literature to be made of aluminium 


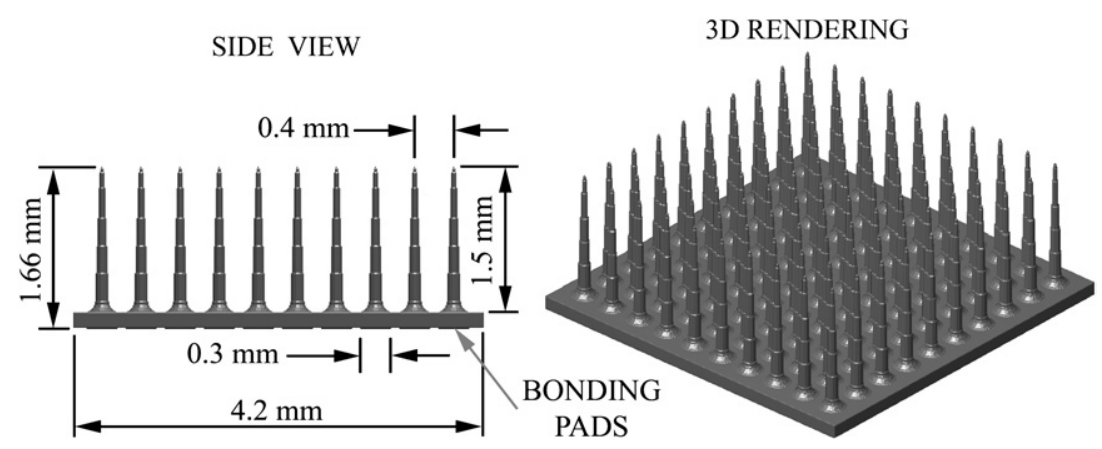

Figure 1. Simplified three-dimensional model used to estimate the magnetic field perturbation produced by the 'Utah probe'. The square bonding pads, located at the bottom of the assembly, have dimensions of $300 \times 300 \mu \mathrm{m}^{2}$.

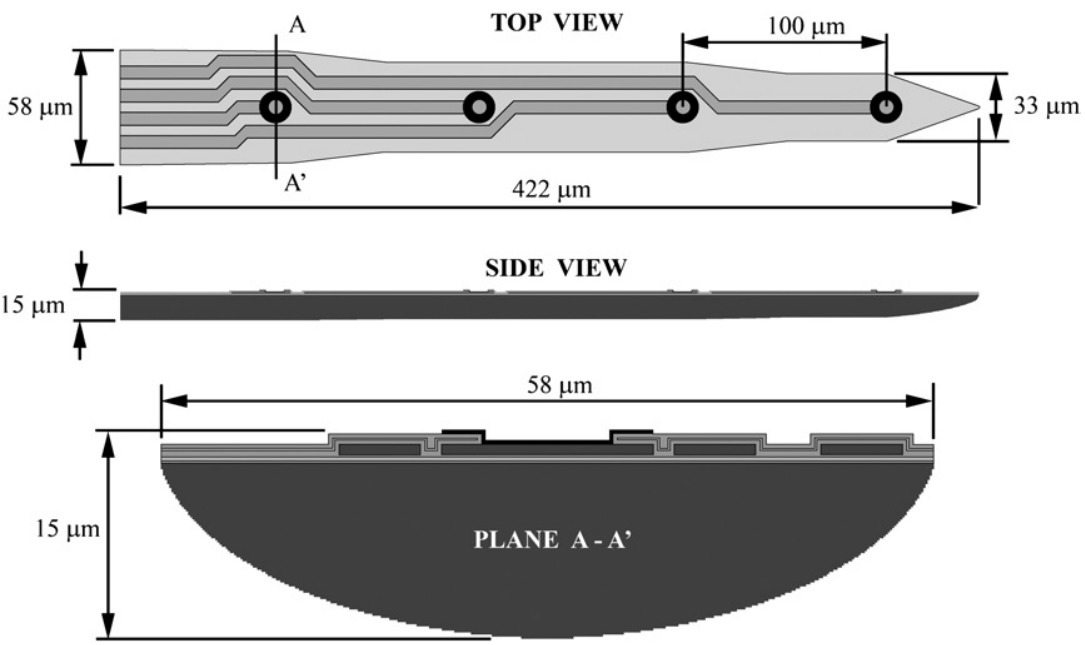

Figure 2. Model of a single-shank 'Michigan probe'. The first site, closer to the tip of the electrode, as well as the third site, were simulated as recording channels and therefore made of iridium $(\chi=37.66)$. The second and fourth sites were considered for neural stimulation, thus made of iridium-oxide $\left(\mathrm{IrO}_{2}, \chi=146.89\right)$. The $\mathrm{A}-\mathrm{A}^{\prime}$ plane is not displayed at the same scale used in the top and side views. The 3D model was obtained simulating the deep boron diffusion process normally used to form the body of the electrode (Finn and LoPresti (2003), chapter 7).

(Al, $\chi=20.7 \mathrm{ppm}$ ) (Campbell et al 1991) or platinum (Nordhausen et al 1996) and, therefore, we decided to simulate both cases.

(8) A moderately detailed model of a single-shank silicon microelectrode, known as the 'Michigan probe', developed at the University of Michigan and distributed to neuroscientists by the Centre for Neural Communication Technology (CNCT) (Drake et al 1988, Anderson et al 1989, CNCT 1999), and presented in figure 2.

Table 1 presents the names assigned to each model and the information of field of view (FOV), matrix size and resolution, used to estimate the magnetic field perturbation $\left(\Delta B_{z}\right)$. For all the simulations, we used a magnetic flux density $B_{0}=1 \mathrm{~T}$, and all the discretized models were surrounded by water $(\chi=-9.05 \mathrm{ppm})$ covering a $3 \mathrm{D}$ field of view (FOV) big enough to reach a constant magnetic flux density, at the periphery, close to the value dictated 
Table 1. Name of models, field of view, matrix size and resolution used to estimate the magnetic field perturbations produced by common neural recording/stimulating microelectrodes.

\begin{tabular}{lllll}
\hline No. & Name & FOV $\left(\mu \mathrm{m}^{3}\right)^{\mathrm{a}}$ & Matrix size & Resolution $\left(\mu \mathrm{m}^{3}\right)^{\mathrm{a}}$ \\
\hline 1 & Inf-Pt & $204.8 \times 204.8$ & $8192 \times 8192$ & $0.025 \times 0.025$ \\
2 & Inf-W & $204.8 \times 204.8$ & $8192 \times 8192$ & $0.025 \times 0.025$ \\
3 & Inf-Si-Au-Pt & $204.8 \times 204.8$ & $8192 \times 8192$ & $0.025 \times 0.025$ \\
4 & Pt-tip & $512 \times 512 \times 1024$ & $1024 \times 1024 \times 2048$ & $0.5 \times 0.5 \times 0.5$ \\
5 & W-tip & $512 \times 512 \times 1024$ & $1024 \times 1024 \times 2048$ & $0.5 \times 0.5 \times 0.5$ \\
6 & Si-Au-Pt-tip & $512 \times 512 \times 1024$ & $1024 \times 1024 \times 2048$ & $0.5 \times 0.5 \times 0.5$ \\
7 & Utah array & $10240 \times 10240 \times 10240$ & $1024 \times 1024 \times 1024$ & $10 \times 10 \times 10$ \\
8 & Michigan probe & $614.4 \times 153.6 \times 76.8$ & $4096 \times 1024 \times 512$ & $0.15 \times 0.15 \times 0.15$ \\
\hline${ }^{a} \mu \mathrm{m}^{2}$ for 2D models. & & &
\end{tabular}

by the Lorentzian sphere correction (Haacke et al (1999), chapter 25, pp 753-7). This way, we minimized aliasing artefacts produced by the periodic nature of the Fourier-based method used to estimate $\Delta B_{z}$ for models $3-8$, and that is explained in the following section. All the simulations were performed using Matlab V7 (R14) Service Pack 3 (The MathWorks, Inc., Natick, MA, USA).

Models 1 and 2 are discretized models of an infinite circular cylinder of $25 \mu \mathrm{m}$ diameter and represent the body, excluding the tip, of commonly used microwires. Model 3 was defined in order to have a direct comparison, by using the same geometry, between metallic microwires and silicon-based microelectrodes, and to analyse the effects of metallization layers applied at the surface of some silicon-based microelectrodes. The infinite nature of these models allowed us to use a 2D specification, instead of 3D, of the magnetic susceptibility distribution, with very high spatial resolution. Models 4-6 complement the previous three models by having just the tip of the microelectrodes, but this time with a limited resolution since we required a $3 \mathrm{D}$ model and the computational time and memory requirements were much higher. Similarly, the limited resolution used for the Utah array (model 7) was because it is a complete $3 \mathrm{D}$ representation that covers a volume of $4.2 \times 4.2 \times 1.66 \mathrm{~mm}^{3}$, which is much bigger than the other models, and therefore demanded considerable computational time and disk space. For the Michigan probe (model 8), we use a $422 \mu \mathrm{m}$ section of a single-shank silicon microelectrode with four channels with a surface area of $177 \mu \mathrm{m}^{2}$ each, and spaced $100 \mu \mathrm{m}$ centre to centre.

For those cases where the model had a symmetry axis and finite length, the magnetic field perturbation was estimated for only two orthogonal orientations, one of them being the symmetry axis. If the length of the model was infinite, as those cases that emulate the main body of microwires, then $\Delta B_{z}$ was only estimated for the condition where the magnetic field is perpendicular to the symmetry axis, because, if they are aligned, there is no magnetic field perturbation outside the cylindrical object. For all other cases, the whole set of three orthogonal orientations of the magnetic field with respect to the object was analysed.

\subsection{Magnetic field perturbation of a single material infinitely long circular cylinder}

The magnetic field perturbation of this simple geometry, including the Lorentzian sphere correction, has been presented and analysed elsewhere (Haacke et al 1999, Schenck 1996) and therefore we only present the closed-form solution, in cylindrical coordinates (1), where $\chi_{e}$ and $\chi_{i}$ are the magnetic susceptibility outside and inside the cylinder, respectively, $\Delta \chi=\chi_{i}-\chi_{e}, B_{0}$ is the applied magnetic field, $a$ is the radius of the cylinder, $(\rho, \phi)$ are the cylindrical coordinates of a particular point in space, and $\theta$ is the angle between the 
symmetry axis of the cylinder and the applied magnetic field.

$$
\Delta B_{z}= \begin{cases}\frac{\Delta \chi}{6}\left(3 \cos ^{2} \theta-1\right) B_{0}+\frac{1}{3} \chi_{e} B_{0} & : \quad \rho<a \\ \frac{\Delta \chi}{2} \frac{a^{2}}{\rho^{2}} \sin ^{2} \theta \cos 2 \phi B_{0}+\frac{1}{3} \chi_{e} B_{0}: & : \quad \rho \geqslant a .\end{cases}
$$

\subsection{Magnetic field perturbation of arbitrary-shaped finite objects}

As mentioned before, we used a Fourier-based method, proposed by Marques and Bowtell (2005), to estimate the magnetic field perturbation $\Delta B_{z}(\mathbf{r})$ produced by a microelectrode, immersed in a tissue-like material, and under the effect of a uniform magnetic field. We used a homogeneous magnetic flux density $B_{0}=1 \mathrm{~T}$ applied along the $z$-direction, and then we estimated the $z$-component of the induced magnetization $M_{z}(\mathbf{r})(2)$ and its corresponding value in $k$-space $M_{z}(\mathbf{k})$ by using a 3D Fourier transform (3).

$$
\begin{aligned}
& M_{z}(\mathbf{r})=\chi(\mathbf{r}) \frac{B_{0}}{\mu_{0}(1+\chi(\mathbf{r}))} \\
& M_{z}(\mathbf{k})=\mathscr{F}_{3 \mathbf{D}}\left\{M_{z}(\mathbf{r})\right\} .
\end{aligned}
$$

The magnetic field perturbation in $k$-space $\Delta B_{z}(\mathbf{k})$ is defined in (4), where $\mu_{0}$ is the permeability, $\overrightarrow{\mathbf{k}}$ is a vector defining a particular point in $k$-space, $\beta$ is the angle between the direction of the main magnetic field $\overrightarrow{\mathbf{B}}_{0}$ and $\overrightarrow{\mathbf{k}}$, and $(\phi, \theta, \psi)$ are the Euler's angles that define any rotation of $\overrightarrow{\mathbf{B}}_{0}$ with respect to the object. Finally we use an inverse 3D Fourier transform to obtain the magnetic field perturbation in the space domain $\Delta B_{z}(\mathbf{r})(6)$.

$$
\begin{aligned}
& \Delta B_{z}(\mathbf{k})=-\frac{\mu_{0} M_{z}(\mathbf{k})}{3}\left(3 \cos ^{2} \beta-1\right) \\
& \cos \beta=\frac{-k_{x} \sin \theta+k_{y} \cos \theta \sin \psi+k_{z} \cos \theta \cos \psi}{\sqrt{k_{x}^{2}+k_{y}^{2}+k_{z}^{2}}} \\
& \Delta B_{z}(\mathbf{r})=\mathscr{F}_{3 \mathbf{D}}^{-1}\left\{\Delta B_{z}(\mathbf{k})\right\} .
\end{aligned}
$$

We used the ' $x y z$ ' convention of the Euler's angles $(\phi, \theta, \psi)$, where $\phi$ defines a rotation around the $z$-axis, $\theta$ around the $y$-axis and $\psi$ around the $x$-axis. The compound rotation is applied in that order, i.e. first the rotation around $z$, then the rotation around $y$ and finally the rotation around $x$, where each rotation is considered positive if it is clockwise when looking down the axis towards the origin.

\subsection{MRI phantoms and imaging parameters}

For all our MRI experiments we used an Oxford 2.0 T horizontal magnet, with a clear bore diameter of $31 \mathrm{~cm}$, equipped with Acustar S-180 gradients of $18 \mathrm{~cm}$ bore diameter, driven to $70 \mathrm{mT} \mathrm{m}^{-1}$, using a Varian Unity Inova imaging platform, and a solenoid coil that closely matched the sampled volume.

In order to have a direct comparison between metallic and silicon-based microelectrodes, we used three different types of microelectrodes: a $25 \mu \mathrm{m}$ diameter Pt-Ir (90\%-10\%) microwire, a tungsten wire that tapered from $370 \mu \mathrm{m}$ at the body to approximately $15 \mu \mathrm{m}$ at the tip, and a 16-channel single-shank Michigan microelectrode, as shown in figure 3. Each 


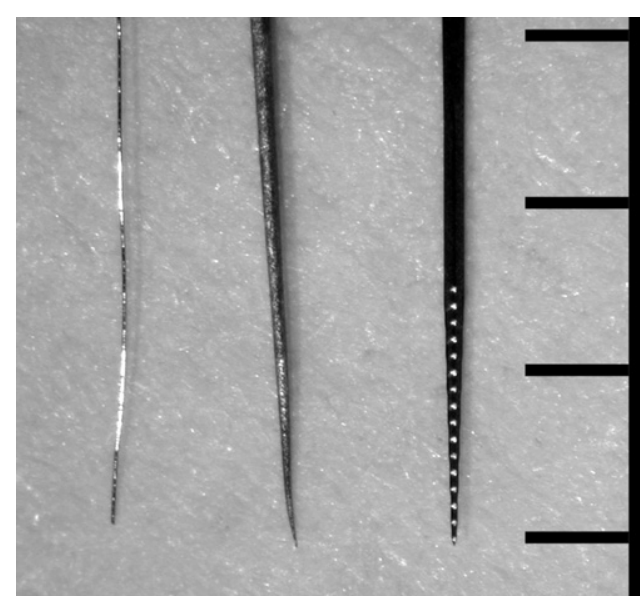

Figure 3. Microelectrodes used for the MRI test. From left to right, platinum-iridium (Pt 90\%-Ir $10 \%$ ), tungsten and single-shank 16-channel silicon microelectrode. An approximate length of $3 \mathrm{~mm}$ is shown for each electrode (scale at the right in $\mathrm{mm}$ ) and for the silicon microelectrode the channel spacing is $100 \mu \mathrm{m}$.

type of microelectrode was attached to a plastic rod and the assembly was then introduced into a glass test tube previously filled with a $3 \mathrm{mM}$ doped water (Gadiodiamide, Omniscan Labs), to reduce $T_{1}$ to approximately $300 \mathrm{~ms}$. The samples were positioned perpendicular to the magnetic field, and we used a 3D gradient echo (GE) pulse sequence with the following imaging parameters: FOV $=1.5 \times 1.5 \times 1.5 \mathrm{~cm}^{3}$, slab thickness $(\mathrm{THK})=1.2 \mathrm{~cm}$, repetition time $(\mathrm{TR})=100 \mathrm{~ms}$, echo time $(\mathrm{TE})=15 \mathrm{~ms}, 4$ averages $(\mathrm{NAV})$, bandwidth $(\mathrm{BW})$ of $15 \mathrm{kHz}$, and a matrix size of $150 \times 150 \times 150$ points.

\section{Results}

The following group of images presents the estimated magnetic field perturbations for all our simulations. For the images obtained from models of infinite length, the magnetic field values inside the cylinders and at the periphery of the FOV have been preserved, and therefore include the Lorentzian correction. This can be observed on the asymmetric colour bars of the pertinent figures. For the other models, with finite spatial extent, the Lorentzian correction outside the electrodes has been removed. Given that the magnetic field inside the objects is not relevant to our analysis, it has been set equal to the background magnetic field and therefore the magnetic field at the periphery and inside the microelectrodes appears to be zero. Additionally, the colour scale of the corresponding images has been limited to $[-1,1]$ ppm and therefore, values of $\Delta B_{z} \leqslant-1 \mathrm{ppm}$ appear black, and values of $\Delta B_{z} \geqslant 1 \mathrm{ppm}$ are saturated in white. In general, the presented images are smaller than the whole FOV used for the simulations, but they include the main portions of magnetic field variations, and exclude some artefacts produced by the spatial truncation of the models. These modifications improve the visual comparisons of the different results, by using a normalized grey scale, and facilitate the analyses.

\subsection{Main body of microelectrodes}

Figure 4 summarizes the results of infinitely long circular platinum and tungsten microwires, as well as an ideal model purely made of silicon. As observed, metallic microwires produce 

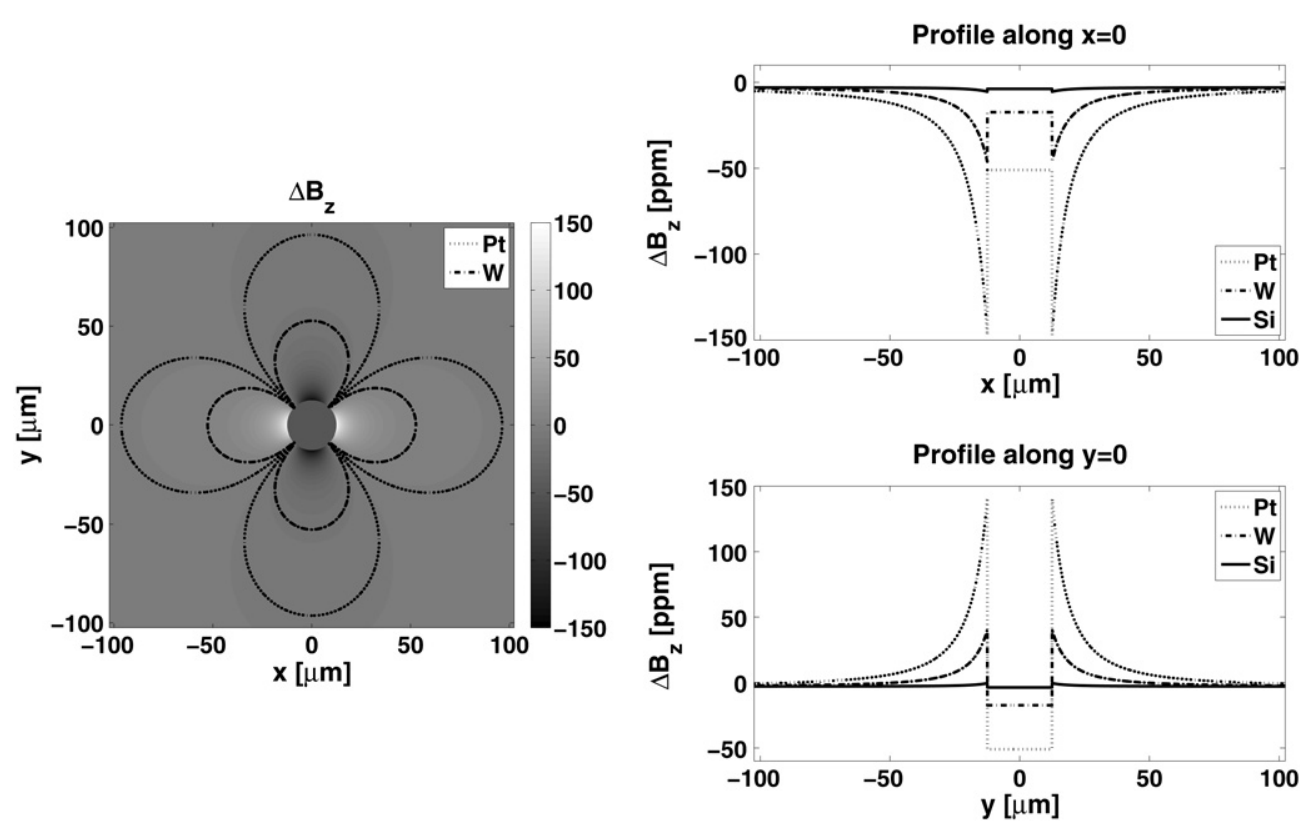

Figure 4. Comparison of the magnetic field perturbations of infinitely long platinum and tungsten microwires and an ideal model, with similar geometry, of a pure silicon solid cylinder. The contour lines over the axial view of $\Delta B_{z}$ represent the distance at which the magnetic field perturbation of the metallic microwires reaches the same value as the maximum field perturbation produced by the silicon model. The colour bar, indicating the magnetic field perturbation in ppm, corresponds to $\mathrm{Pt}$ and can be scaled to $\mathrm{W}$ or $\mathrm{Si}$ using the maximum and minimum values of the profiles, along $x=0$ and $y=0$, presented at the right graphs.

significant field perturbations whereas the silicon model produces barely noticeable changes in the surrounding media. The maximum change of the magnetic field, with respect to the background, occurs at the surface of the cylinders, being $144.025 \mathrm{ppm}$ for Pt, $43.125 \mathrm{ppm}$ for $\mathrm{W}$ and $2.425 \mathrm{ppm}$ for Si. Interestingly, the maximum distance for the metallic microwires to achieve a field perturbation equal to the maximum perturbation of the Si model are $96.35 \mu \mathrm{m}$ for $\mathrm{Pt}$, and $52.7 \mu \mathrm{m}$ for $\mathrm{W}$, a factor of 7.7 and 4.2, respectively, with respect to the original radius of the objects.

\subsection{Metallization layers}

Figure 5 presents the effects of gold and platinum layers added to a solid infinite cylindrical core, purely made of silicon. Given the discretization of the 3D models and the method used to estimate $\Delta B_{z}$, some ringing artefacts occur at the edges of each layer. In addition, the diamagnetic and paramagnetic nature of adjacent materials produced significant polarity changes of the magnetic flux density inside the metallic layers. In general, large spikes from the ringing artefact were observed between 3 to 5 pixels away from an edge, which correspond to an average distance of $0.1 \mu \mathrm{m}$ and therefore were too small to be significant for our analysis.

\subsection{Tip of microelectrodes}

Figures 6 and 7 summarize the results obtained for the Pt-Tip, W-Tip and Si-Au-Pt-Tip models for two different orientations of the magnetic field with respect to the microelectrodes. 


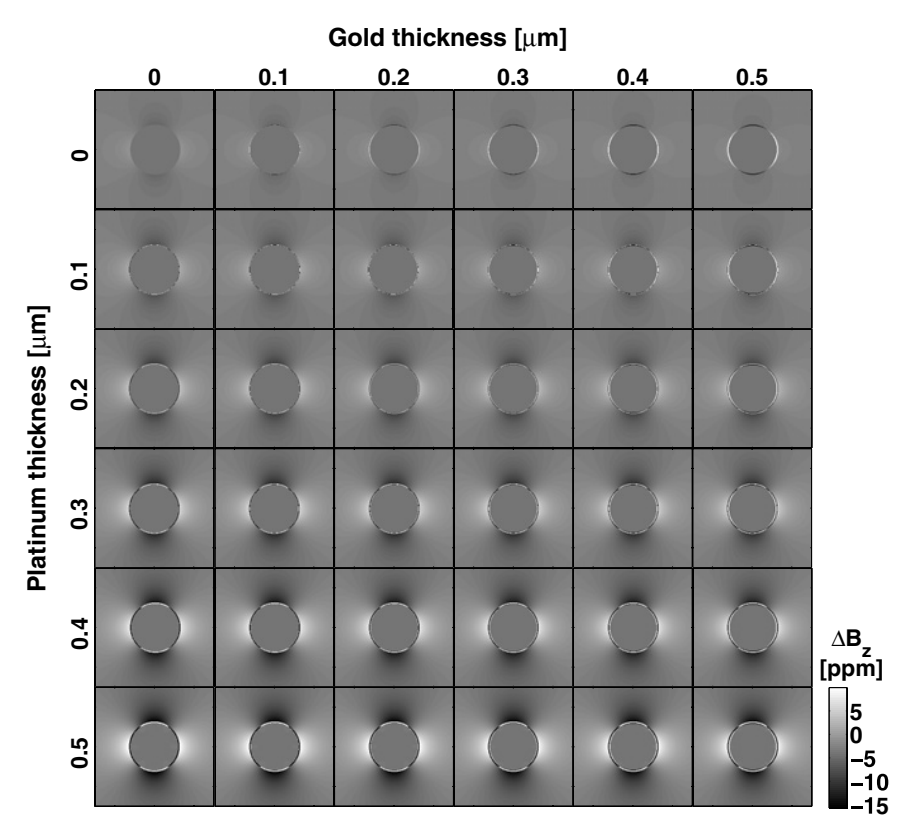

Figure 5. Analysis on the magnetic field perturbations produced by metallization layers added over a silicon core. The colour scale, presented at the lower right corner, represents the magnetic field perturbation in ppm, and it was limited to the values encountered outside the cylinder. Therefore the large field variation found inside the cylinder and the metallic layers, which are not relevant for our analysis, are saturated and appear completely white or black. The range of the colour scale was determined by the worst case, which corresponds to a Pt layer of $5 \mu \mathrm{m}$ thick and no gold.

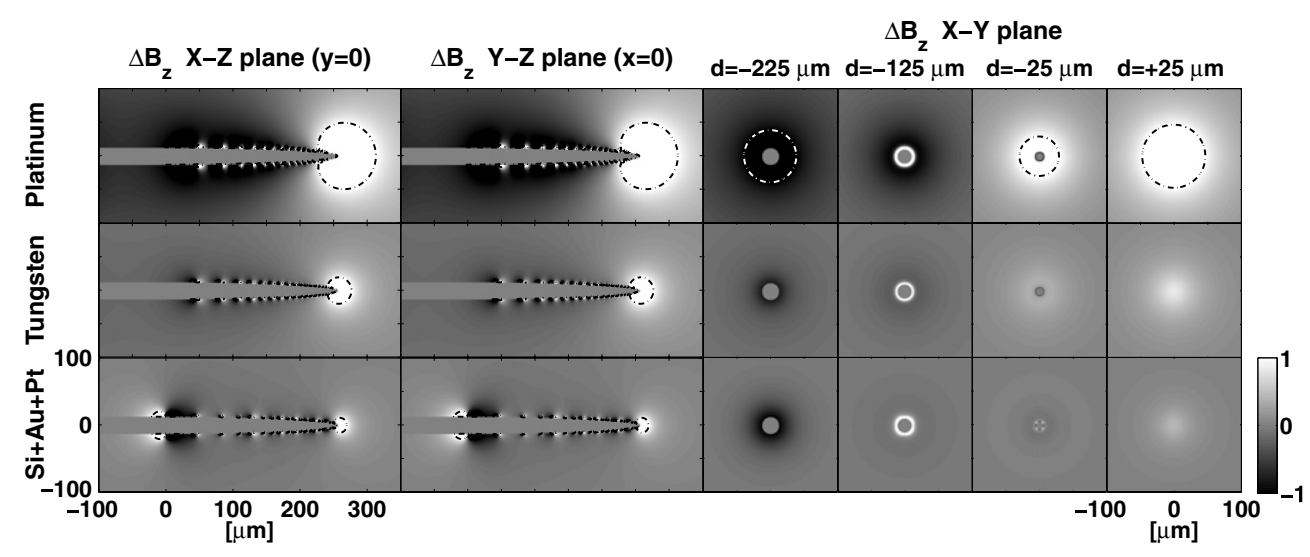

Figure 6. Simulations of the magnetic field perturbations produced at the tip of tapered wire microelectrodes when the magnetic field is aligned to the symmetry axis. The contour lines indicate the points where $\Delta B_{z}$ is $\pm 1 \mathrm{ppm}$ with respect to the background, and the distance ' $d$ ' is measured from the tip of the electrode.

For the case where the magnetic field is parallel to the symmetry axis, the discretization of the models produces artefactual field variations at every point where the electrode narrows down a single step. Nevertheless, the model nicely predicts the 'needle-tip' artefact reported 


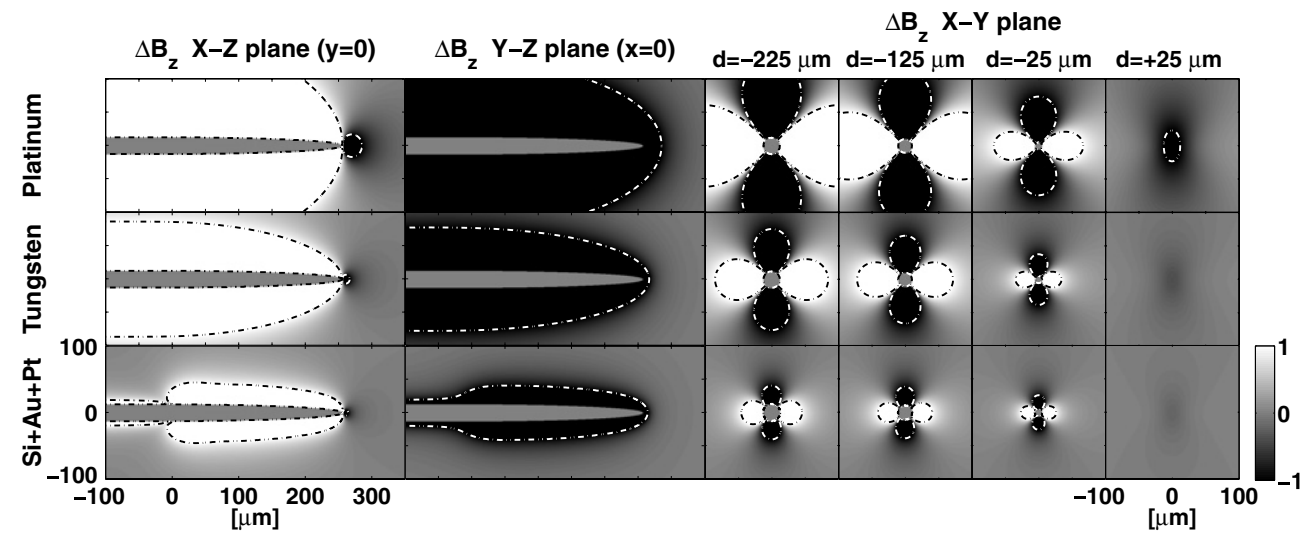

Figure 7. Simulations of the magnetic field perturbations produced at the tip of tapered wire microelectrodes when the magnetic field is perpendicular to the symmetry axis and aligned with the $x$-axis. Similarly to figure 6 , the contour lines indicate the points where $\Delta B_{z}$ is $\pm 1 \mathrm{ppm}$ with respect to the background and ' $d$ ' is measured to the right $(d>0)$ or to the left $(d<0)$ of the tip of the electrode.

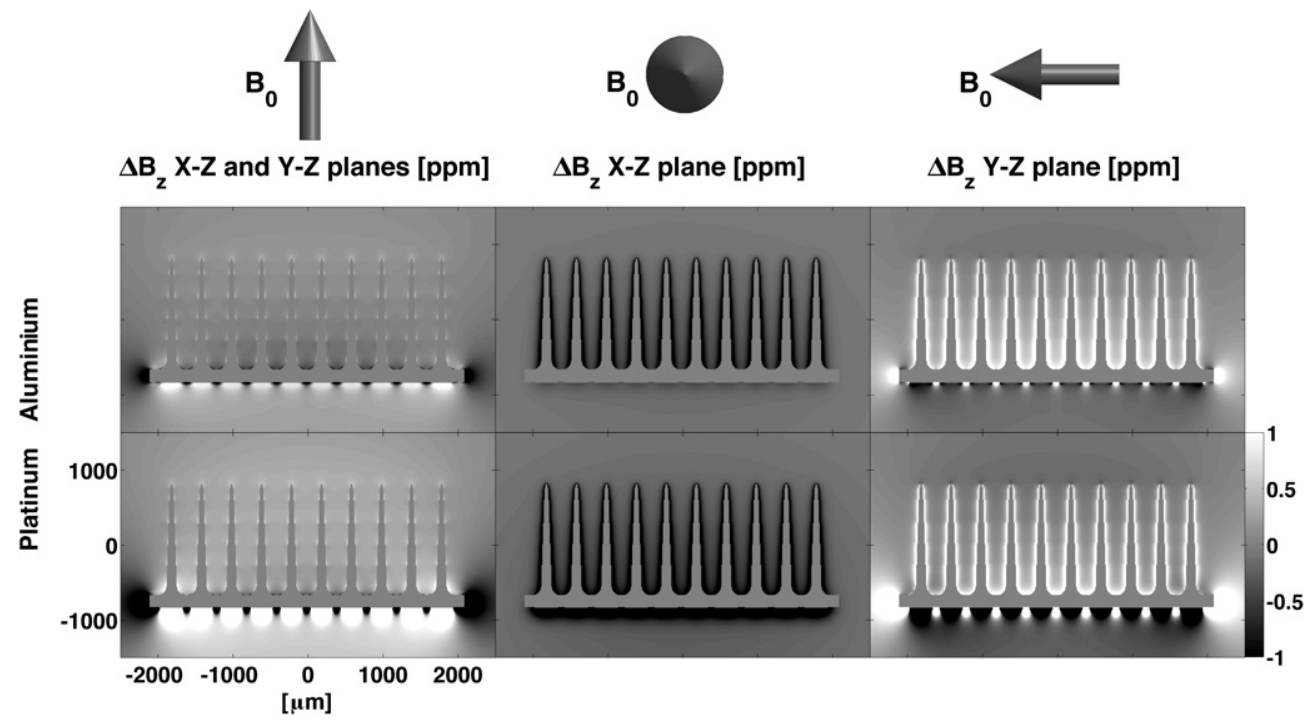

Figure 8. Simulations of the magnetic field perturbations produced by simplified models of the Utah array. The top row corresponds to a three-dimensional model that uses bonding pads made of $\mathrm{Al}$ whereas the bottom row presents the case of bonding pads made of Pt.

in the literature (Liu et al 2001), and observed as a significant perturbation of the magnetic field that occurs right at the tip of needles and cannulas used in MRI-guided surgeries, and also observed in wire microelectrodes (Matsui et al 2005).

\subsection{The Utah probe}

Figure 8 presents the magnetic field perturbation of the simplified model of the Utah probe. Given that the metallization layers at the tip of the shanks were omitted, the principal sources of 


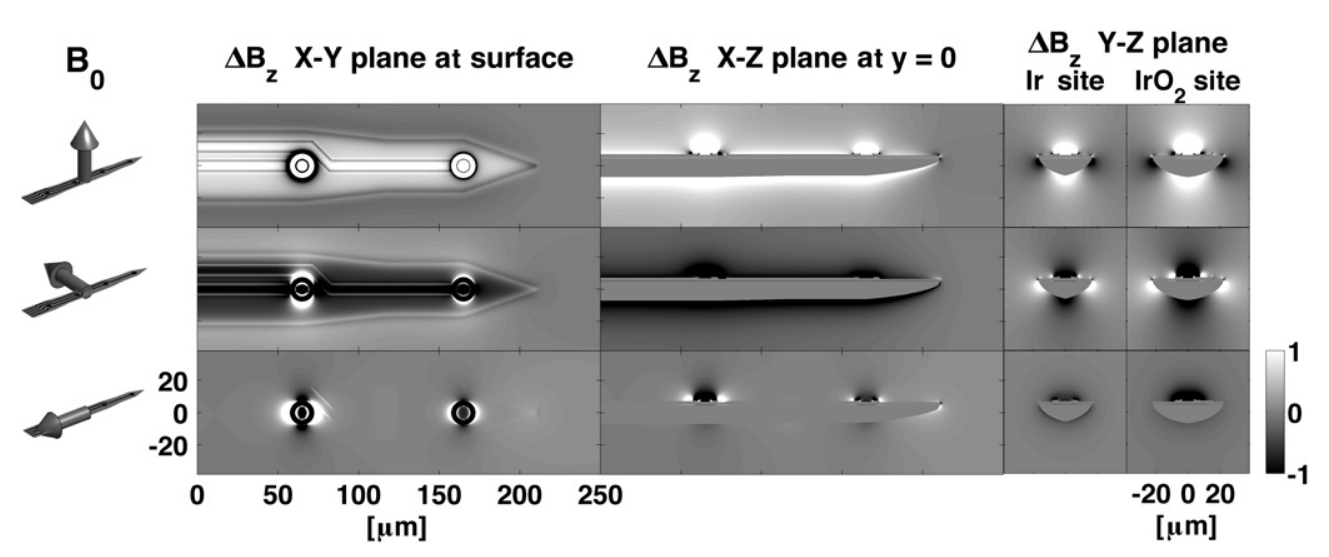

Figure 9. Simulations of the magnetic field perturbations produced by a section of a single-shank Michigan probe. Only the first two channels, out of four, are shown; the site closer to the tip is made of iridium, as used for neural recordings, and the second one is made of $\mathrm{IrO}_{2}$, commonly used for neural stimulation.

magnetic field perturbations are the bonding pads at the bottom of the assembly. As expected, $\mathrm{Pt}$ produces perturbation of larger spatial extent, compared to $\mathrm{Al}$.

\subsection{The Michigan probe}

Figure 9 presents the magnetic field perturbations of a section of a single-shank four-channel microelectrode. As can be seen, the magnetic field perturbations are very small compared to the previous models, even for the sites where $\mathrm{IrO}_{2}$ is used. The needle-tip artefact is also present, although with a much smaller spatial extent and magnitude.

\subsection{Qualitative MRI comparison}

Figure 10 presents a qualitative MRI comparison of the platinum-iridium, tungsten and silicon microelectrode tested at $2 \mathrm{~T}$. As presented by the minimum intensity projections (mIP), the PtIr and tungsten microwires produced significant signal loss that extends several micrometres away from the body of the electrodes. On the other hand, the Michigan probe only produces a partial volume effect on the voxels occupied by the electrode. As a rough estimate, using a voxel size of $100 \times 100 \times 100 \mu \mathrm{m}^{3}$, we can observe that the $25 \mu \mathrm{m} \mathrm{Pt}-\mathrm{Ir}$ microwire is transformed to a void of approximately $500 \mu \mathrm{m}$, a factor of 20 under the specific testing conditions. For the tungsten microwire, that factor is approximately 13 at the tip, and for the silicon microelectrode, using the thickness of $15 \mu \mathrm{m}$ as a reference, the factor is only about 7 .

\section{Discussion}

The presented results show that metallic microwires produce significant magnetic field perturbations that compromise the anatomical or functional information of MR images in regions surrounding the microelectrodes. The severity of the artefacts depends on several factors such as magnetic field strength, position and orientation of the electrode, type of microelectrode, pulse sequence, and echo time, among others. 


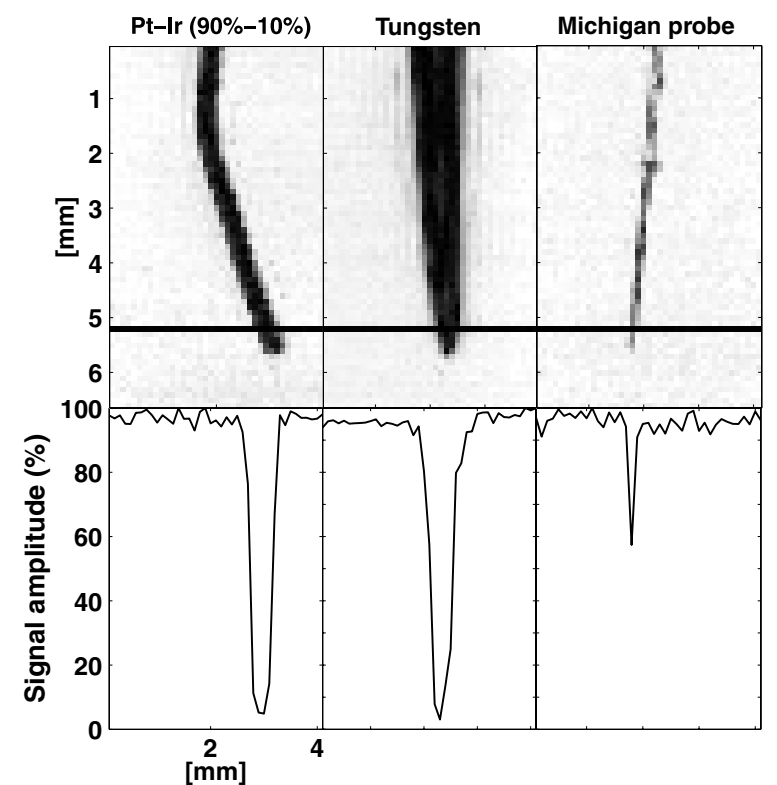

Figure 10. Minimum intensity projections (top) and signal intensity profiles (bottom) of MR images from Pt-Ir, tungsten and silicon microelectrodes. The profiles presented in the bottom row correspond to the signal intensity across the horizontal black lines of the mIPs, which are approximately $500 \mu \mathrm{m}$ away from the tip of the electrodes.

For example, the needle-tip artefact is more prominent when the electrode is aligned with the main magnetic field and changes its shape, polarity and spatial extent as the alignment varies, until it forms a smaller spheroid when the magnetic field is perpendicular to the electrode. Based on our simulations, a $1 \mathrm{ppm}$ needle-tip artefact at $1 \mathrm{~T}$, for Pt microwires, is delimited by a quasi-spherical surface of about $100 \mu \mathrm{m}$ in diameter, and almost centred at the tip of the electrode. This region easily covers the volume of neural activity detected or induced by the electrode. For tungsten microwires, that diameter is reduced to approximately $40 \mu \mathrm{m}$.

Importantly, we know that the magnitude of the magnetic field perturbations linearly increases as the static magnetic field $B_{0}$ is increased. For example, if we increase the main magnetic field from 1 to $3 \mathrm{~T}$, the $100 \mu \mathrm{m}$ spheroidal artefact, produced by the Pt microwire, would enclose a region of $\Delta B_{z} \geqslant 3 \mathrm{ppm}$, which in the lower boundary is close to the chemical shift of fat with respect to water. Depending on the imaging protocol, this frequency shift may have significant effects.

As shown in figure 7, when $B_{0}$ is perpendicular to the wire microelectrodes, the magnetic field perturbation is more prominent towards the sides of the electrode, i.e. perpendicular to the longitudinal walls of the microwire. Taking as a reference the symmetry axis, the $\pm 1 \mathrm{ppm}$ boundary at $1 \mathrm{~T}$ for Pt microwires starts at about $25 \mu \mathrm{m}$ in front of the tip and rapidly grows to distances grater than $100 \mu \mathrm{m}$ as we move towards the body of the electrode. For $\mathrm{W}$, this boundary remains at a distance below $80 \mu \mathrm{m}$ for most of the body of the microwire and falls to about $10 \mu \mathrm{m}$ at points right ahead of the tip.

The lower row of figure 7 nicely presents the effects of metallization layers added to a silicon core. As observed, the $\pm 1 \mathrm{ppm}$ boundary at $1 \mathrm{~T}$ produced by the silicon core alone is relatively small $(z<0)$, and abruptly increases to a radius of about $50 \mu \mathrm{m}$ as soon as the added $\mathrm{Pt}$ and $\mathrm{Au} 0.5 \mu \mathrm{m}$ layers are present $(z>0)$. On the same row of images, the transverse 
planes at different distance ' $d$ ' show that the $1 \mathrm{ppm}$ field perturbation practically triples the diameter of the microelectrode.

This effect should be taken in consideration when analysing the results of the Utah probe, presented in figure 8 . Not only the bonding pads, at the base of the 100-shank array, produce significant artefacts but also the metallization layers added to each shank contribute to the magnetic field perturbations. Furthermore, the relatively close proximity of the 100 shanks would have an additive effect of $\Delta B_{z}$ on the interstitial spaces between the shanks, increasing the overall magnetic field perturbation.

Of all our simulations, the Michigan probe presents the best characteristics of MR compatibility, presenting a very small MR signature. As observed in figure 9, the maximum field variations occur at stimulating channels, affecting regions in close proximity to the sites. Absolute magnetic field perturbation in excess of $1 \mathrm{ppm}$ at $1 \mathrm{~T}$ does not exceed distances of $10 \mu \mathrm{m}$ away from the surface of the electrode. In addition, the needle-tip artefact is very small.

Even though the Michigan probe seems to be MR-friendly, there are some problems that need to be solved before this particular technology can be used for simultaneous neural recordings or stimulation and fMRI studies. In particular, the very high impedance of the recording electrodes, which is in the range of 1 to $2 \mathrm{M} \Omega$ at $1 \mathrm{KHz}$, in combination with the high gain of recording amplifiers, makes the electrode very sensitive to MR-induced currents. For instance, a $1 \mu \mathrm{A}$ of induced current on a $1 \mathrm{M} \Omega$ electrode would produce a $1 \mathrm{~V}$ offset voltage that can saturate an input amplifier commonly used for this type of probe. Another problem is the short life span of the connectors currently used in MR-compatible probes (MartínezSantiesteban et al 2006), which have a life span of only ten connection/disconnection cycles.

In theory, one should be able to design a MR-compatible device that does not produce significant magnetic field perturbations, by choosing the right type, amount and mixture of materials. This idea has been successfully tested for simple cylindrical copper conductors coated with rhodium, for the fabrication of NMR RF probes (Zelaya et al 1995). Basically, they use the closed-form equations that define the magnetic field perturbations produced by two concentric and adjacent infinite cylinders, one being a solid core of radius $a$ and magnetic susceptibility $\chi_{\text {core }}$, and the other one the coating material of thickness $\delta$ and magnetic susceptibility $\chi_{\text {coating. }}$. The total radius $(b=a+\delta)$ required to achieve zero magnetic field perturbation on the surrounding media, with magnetic susceptibility $\chi_{e}$, is given by (7).

$$
b=a \sqrt{\frac{\chi_{\text {coating }}-\chi_{\text {core }}}{\chi_{\text {coating }}-\chi_{e}}} .
$$

An attempt to use the same idea for simultaneous electrical stimulation and fMRI is presented in Alwatban et al (2002). Unfortunately, we believe that they miscalculated the thickness of the coating and also matched to an external condition of zero magnetic susceptibility, instead of matching to the magnetic susceptibility of air, bone or tissue, which are the three interfaces encountered in that particular experiment.

Even though the concept of achieving MR compatibility using a mixture of diamagnetic and paramagnetic materials is very attractive, in practice, this idea might be difficult to implement. Although we can find paramagnetic materials with almost any value of magnetic susceptibility greater than zero, the range of values for diamagnetic materials is bounded to $\chi=-1$ (Schenck 1996) and in practice, there are relatively few useful materials with $\chi \leqslant-50 \mathrm{ppm}$. This implies the need of larger amounts of diamagnetic materials to counterbalance the paramagnetic ones. This effect can be observed on figure 11. Given that gold is more diamagnetic than water, soft tissue and silicon, thicker layers of gold counteract the perturbation produced by $\mathrm{Si}$ and $\mathrm{Pt}$. Unfortunately, the paramagnetic nature of $\mathrm{Pt}$ is a lot 


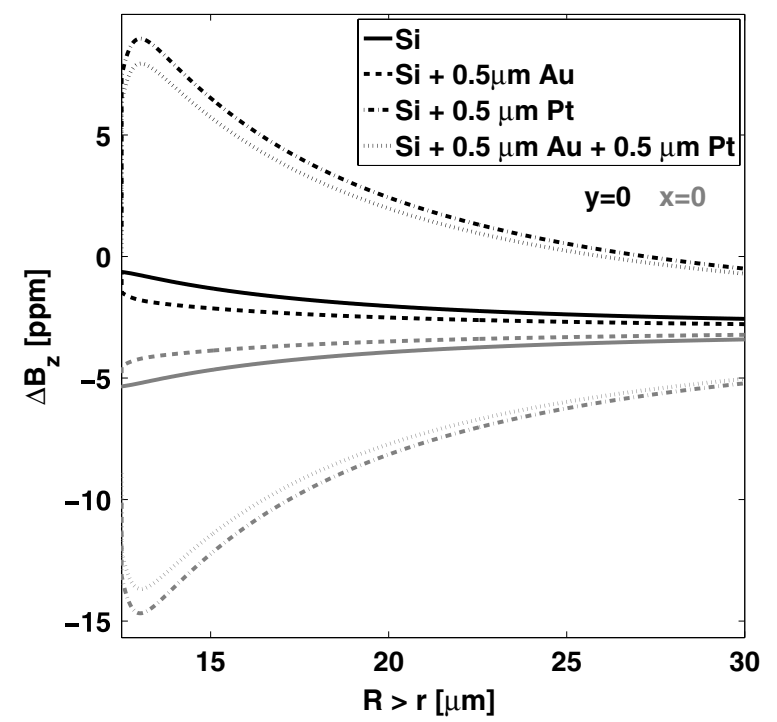

Figure 11. Main profiles of $\Delta B_{z}$ for different thickness of metallization layers, obtained from figure 5 .

bigger than the diamagnetism of gold or silicon and therefore the magnetic field perturbation is dominated by Pt.

Similarly, following the model of an infinite cylinder, a Pt microwire of $25 \mu \mathrm{m}$ diameter would require a gold coating of $31.8 \mu \mathrm{m}$, increasing the final size of the microwire 3.54 times the original diameter. For tungsten, a gold coating of $13.89 \mu \mathrm{m}$ would be required, increasing the total diameter by a factor of 2.1. Obviously, we may think of materials with different and larger diamagnetic properties, to create smaller final diameters, but then biocompatibility may become an issue. If we invert the order of the materials, i.e. a core of gold with a coat of platinum or tungsten, a golden microwire with $25 \mu \mathrm{m}$ diameter would require a coating of $530 \mathrm{~nm}$ of Pt or $1.69 \mu \mathrm{m}$ of W. For any situation, the mechanical, chemical, electrical and biocompatibility characteristics of the susceptibility-matched microwire would change, perhaps significantly, with respect to the original microelectrode.

Finding the right materials and thickness of matching layers could be even more problematic for more complex geometries, such as the Utah or Michigan probes, although the possibility is there, waiting to be exploited in the near future.

Another important artefact in MRI occurs when magnetic field perturbations produce imperfections in the excited slice. If $\left|\Delta B_{z}\right|$ is sufficiently high in particular regions within the slice, then the protons within those regions will not be fully excited. This is due to the fact that they fall outside the excitation bandwidth of the applied RF pulse. At the same time, regions outside the desired slice may be excited and appear to be inside the slice. For example, a $3 \mathrm{~ms} 5$-lobe sinc-shaped RF pulse would have an approximate bandwidth of $2 \mathrm{kHz}$ and would not fully excite regions with magnetic field perturbations greater than $16 \mathrm{ppm}$ at 3 T. As observed in figure 4 , after applying a factor of 3 to $\Delta B_{z}$, magnetic field perturbations in excess of $16 \mathrm{ppm}$ occur at a significant distance from the tissue-microelectrode boundary of metallic microelectrodes. Furthermore, positional errors in the frequency-encode direction would become more apparent on those regions that fall within the excitation bandwidth and have sufficient frequency shifts per pixel. Imaging protocols with relatively low bandwidth or long readout intervals would be particularly vulnerable. For instance, in a blipped EPI 
pulse sequence, the number of pixels shifted due to a magnetic field perturbation $\Delta B$ is given by $n_{\mathrm{pe}}=N_{\mathrm{pe}} \bar{\gamma} \Delta B \Delta \tau_{\mathrm{pe}}$ and $n_{\mathrm{ro}}=N_{\mathrm{ro}} \bar{\gamma} \Delta B \Delta t=\Delta B /\left(G_{\mathrm{ro}} \Delta d_{\mathrm{ro}}\right)$, where the subscripts 'pe' and 'ro' correspond to the phase encode and readout directions, respectively; $n$ is the number of shifted pixels, $N$ is the number of points, $\Delta \tau_{\mathrm{pe}}$ is the time between two successive phase encoding gradients (blips), $\Delta t$ is the time between adjacent sampled points ( 1 /sampling rate), $G$ is the gradient magnitude, $\Delta d$ is the pixel size, and $\bar{\gamma}=42.575 \mathrm{MHz} \mathrm{T}{ }^{-1}$ for hydrogen protons (Haacke et al (1999), chapter 19, pp 546-52). Given that $\Delta \tau_{\mathrm{pe}}>\Delta t$, the distortion in the phase encode direction is more prominent for a given $\Delta B$ and $N_{\mathrm{pe}}=N_{\mathrm{ro}}$. For example, in a $3 \mathrm{~T}$ system with a matrix size of $64 \times 64$, a magnetic field perturbation of $1 \mathrm{ppm}$ and $\Delta \tau_{\mathrm{pe}}=1 \mathrm{~ms}$, we can expect as many as eight shifted pixels in the phase encode direction. In addition, geometric distortions and signal loss would also become more apparent for increased magnetic field perturbations and echo times, respectively. Moreover, the frequency distribution produced by the magnetic field perturbations would reduce the $T_{2}^{*}$ properties of the affected regions, increasing the image artefacts. For long echo times, the Brownian motion of water molecules nearby regions with abrupt magnetic flux density changes may also reduce the apparent $T_{2}$. This effect is produced when protons are excited in the presence of a particular magnetic field and refocused when they have diffused to regions with a significantly different field.

A limitation of the presented study is that, for all the silicon models, we used pure silicon as the base material. According to Campbell et al (1991), the Utah probe uses aluminium as the main doping material, whereas the Michigan probe uses boron-doped silicon for the substrate, and phosphorus-doped polysilicon for recording interconnects (Finn and LoPresti (2003), chapter 7). However, specific information about the magnetic and chemical characteristics of the different doped silicons, used for each technology, is not readily available. Nevertheless, the presented simulations satisfactorily predict the MRI artefacts produced by the tested microelectrodes.

Other technologies, not covered on this work, include glass-coated carbon fibre microelectrodes and polyimide electrodes. The former have been successfully used in simultaneous neural stimulation and fMRI studies (Shyu et al 2004). They produce small but noticeable magnetic field perturbations, and are limited to a single stimulating channel. Unfortunately, the magnetic susceptibility of carbon fibre composites is normally anisotropic (Schenck et al 1995, McClure and Hickman 1982) and highly dependent on manufacturing processes. Therefore the possible MR image artefacts are difficult to predict for arbitrary orientations. In addition, the coating glass and the air trapped between the glass and the carbon fibres may also affect the quality of the images. On the other hand, polyimide microelectrodes are not yet a mature technology, but they seem to be good prospects for MR-friendly microelectrodes.

It is worth noting that the image artefacts presented in figure 10 are specific to the conditions of the experiments, i.e. at $2 \mathrm{~T}$, using a 3D GE pulse sequence, and with a TE of $15 \mathrm{~ms}$, among others. Even though such echo time is relatively short for fMRI experiments, excluding those at very high magnetic fields such as $7 \mathrm{~T}$ or higher, the obtained results give a very good idea of the relative impact in MRI of the different microelectrode technologies. The effects of the magnetic field perturbations produced by each electrode would be significantly more evident at higher magnetic fields, using single-shot fast pulse sequences such as EPI, commonly used for fMRI, or increasing the echo time. Likewise, the artefacts would be reduced for spin echo pulse sequences.

Published results of simultaneous fMRI studies, using single- or multi-shot EPI (4.7 T, resolution $\left.=750 \times 750 \times 2000 \mu \mathrm{m}^{3}, \mathrm{TE}=20 \mathrm{~ms}\right)$, and electrophysiological methods, using Pt-Ir microelectrodes, show a greater correlation between local field potentials (LFP) and the 
BOLD response than those observed between BOLD and single- or muti-unit spiking activity (Logothetis et al 2001). This result is somewhat expected given the relatively slow temporal response and spatial integration of both, the fMRI BOLD effect and LFP, compared to the fast and localized responses of spiking activity. However, in that study, functional images right at the vicinity of the electrode are not reported. Based on our simulations of magnetic field perturbations and MR images of wire microelectrodes, we believe that no useful fMRI BOLD response can be obtained, using EPI, right at the vicinity of Pt-Ir microwires due to the magnitude of the magnetic field perturbation produced by the metallic probes. In the future, given the low magnetic signature of silicon microelectrodes, and perhaps polyimide probes, and advances on high resolution fMRI studies $\left(125 \times 125 \times 750 \mu \mathrm{m}^{3}\right)$ (Logothetis et al 2002), it may be possible to bring more light into the actual relationship between fMRI and spiking activity right in those regions where single- and multi-unit activity does occur.

\section{Conclusions}

As presented in this work, the magnetic field perturbations produced by wire microelectrodes can easily compromise anatomical or functional information in regions where either the neuronal signals are detected or where neural tissue is stimulated by the electrodes. Therefore, the use of this type of device for simultaneous electrophysiology and fMRI is questionable. Importantly, not all silicon-based microelectrodes provide good MR image quality. As observed, the metallization layers added at the tip of some silicon microelectrodes, the geometry, and the materials used to interconnect the electrodes with the external world, can adversely affect the magnetic field in the region where the electrode was implanted.

According to our analysis, Michigan microelectrodes not only provide good channel density, high reproducibility, precise dimensions and other advantages of silicon technology, but also a low magnetic signature that does not significantly affect the quality of MR images.

\section{References}

Alwatban A Z, Ludman C N, Mason S M, O’Donoghue G M, Peters A M and Morris P G 2002 A method for the direct electrical stimulation of the auditory system in deaf subjects: a functional magnetic resonance imaging study J. Magn. Reson. Imaging 16 6-12

Anderson D J, Najafi K, Tanghe S J, Evans D A, Levy K L, Hetke J F, Xue X L, Zappia J J and Wise K D 1989 Batch-fabricated thin-film electrodes for stimulation of the central auditory system IEEE Trans. Biomed. Eng. 36 693-704

Campbell P K, Jones K E, Huber R J, Horch K W and Normann R A 1991 A silicon-based, three-dimensional neural interface: manufacturing processes for an intracortical electrode array IEEE Trans. Biomed. Eng. 38 758-68

CNCT 1999 Passive Multichannel Recording and Stimulating Electrode Arrays. A Catalog of Available Designs (The University of Michigan, Center for Neural Communication Technology)

Drake K L, Wise K D, Farraye J, Anderson D J and Bement S L 1988 Performance of planar multisite microprobes in recording extracellular single-unit intracortical activity IEEE Trans. Biomed. Eng. 35 719-32

Finn W E and LoPresti P G (ed) 2003 Handbook of Neuroprosthetic Methods (New York: CRC Press)

Haacke E M, Brown R W, Thompson M R and Venkatesan R 1999 Magnetic Resonance Imaging. Physical Principles and Sequence Design (New York: Wiley-Liss)

Liu H, Hall W A, Martin A J and Truwit C L 2001 Biopsy needle tip artifact in MR-guided neurosurgery J. Magn. Reson. Imaging 13 16-22

Logothetis N K, Merkle H, Augath M, Trinath T and Uurbil K 2002 Ultra high-resolution fMRI in monkeys with implanted RF coils Neuron 35 227-42

Logothetis N K, Pauls J, Augath M, Trinath T and Oeltermann A 2001 Neurophysiological investigation of the basis of the fMRI signal Nature 412 150-7 
Marques J P and Bowtell R 2005 Application of a Fourier-based method for rapid calculation of field inhomogeneity due to spatial variation of magnetic susceptibility Concepts Magn. Reson. Part B (Magn. Reson. Eng.) 25B 65-78

Martínez-Santiesteban F M, Swanson S D, Noll D C and Anderson D J 2006 Magnetic resonance compatibility of multichannel silicon microelectrode systems for neural recording and stimulation: design criteria, tests, and recommendations IEEE Trans. Biomed. Eng. 53 547-58

Matsui T, Koyano K W, Naya Y, Takeda M, Nakahara K and Miyashita Y 2005 4.7 T MRI-based detection of the tip of an electrode in monkey cortex 2005 Neuroscience meeting, Society for Neuroscience, Program No. 456.10 (Washington, DC)

McClure J W and Hickman B B 1982 Analysis of magnetic susceptibility of carbon fibers Carbon 20 373-8

Nordhausen C T, Maynard E M and Normann R A 1996 Single unit recording capabilities of a 100 microelectrode array Brain Res. 726 129-40

Schenck J F 1996 The role of magnetic susceptibility in magnetic resonance imaging: MRI magnetic compatibility of the first and second kinds Med. Phys. 23 815-50

Schenck J F, Rohling K W, Moriarity T M, Alexander E III and Jolesz F A 1995 Carbon fiber biopsy cannulas for MR-Compatible neurosurgical procedures 3rd Annual Scientific Meeting of the Society of Magnetic Resonance (Nice, Italy) p 1160

Shyu B C, Lin C Y, Sun J J, Chen S L and Chang C 2004 BOLD response to direct thalamic stimulation reveals a functional connection between the medial thalamus and the anterior cingulate cortex in the rat Magn. Reson. Med. 52 47-55

Zelaya F O, Crozier S, Dodd S, McKenna R and Doddrell D 1995 Measurement and compensation of field inhomogeneities caused by differences in magnetic susceptibility J. Magn. Reson. A 115 131-6 